\title{
Lentiviral Vector-Mediated Dopamine D3 Receptor Modulation in the Rat Brain Impairs Alcohol Intake and Ethanol-Induced Conditioned Place Preference
}

\author{
Amine Bahi and Jean-Luc Dreyer
}

\begin{abstract}
Background: It has been reported that dopamine D3 receptor (D3R) knockout mice display similar ethanol (EtOH) consumption compared to wild types. In addition, studies with D3R pharmacological targeting were inconclusive.

Methods: In the current study, we used both gain- and loss-of-function approaches to test the effects of central D3R manipulation on voluntary alcohol intake and EtOH-induced conditioned place preference (CPP) in rats. To this aim, we developed a lentiviral-mediated gene transfer approach to examine whether D3R knockdown (LV-siD3R) or overexpression (LV-D3R) in the nucleus accumbens (NAcc) is sufficient to modulate voluntary alcohol consumption and EtOH-CPP.

Results: Using the standard 2-bottle choice drinking paradigm and an unbiased CPP procedure, our results indicated that, like the D3R selective antagonist SB-277011-A, LV-siD3R attenuated voluntary alcohol consumption. In contrast, LV-D3R increased EtOH intake with no effect on total fluid intake. Similarly, the D3R agonist 7-OH-DPAT also exacerbated EtOH intake. Interestingly, neither pharmacological nor genetic manipulation of D3R activity affected saccharin and quinine consumption and preference. More importantly, we report that LV-siD3R blocked, whereas LV-D3R exacerbated, EtOH-CPP.

Conclusions: These results support the notion that the D3R plays an important role in alcohol reward in rats and suggest that a key threshold range of D3R levels is associated with impaired alcohol consumption. Taken together, these findings demonstrate that the D3R is an essential component of the molecular pathways underlying the reinforcing properties of alcohol. Thus, medications targeting the D3Rs may be beneficial to tackle EtOH abuse and alcoholism in humans.

Key Words: Alcoholism, Conditioned Place Preference, Gene Therapy, Lentivirus, siRNA, TwoBottle Choice.
\end{abstract}

$\mathrm{E}$ VIDENCE HAS ACCUMULATED in recent years implicating the dopaminergic systems in a range of behavioral and neurobiological processes relevant to alcohol craving and relapse (Spanagel and Heilig, 2005; Spanagel and Weiss, 1999). Addicted behavior in alcoholics is characterized by compulsive alcohol-seeking and relapse. Besides environmental and social factors, clearly, genetic factors contribute to compulsive alcohol consumption and relapse. Thus, we have shown recently that decreased dopamine D1 receptor (D1R) expression in the nucleus accumbens (NAcc) leads to reduced ethanol (EtOH)-conditioned rewarding properties and to lower voluntary EtOH consumption,

From the Department of Anatomy (AB), CMHS, United Arab Emirates University, Al Ain, UAE; and Division of Biochemistry (J-LD), Department of Medicine, University of Fribourg, Fribourg, Switzerland.

Reprint requests: Dr. Amine Bahi, Department of Anatomy, College of Medicine \& Health Sciences, United Arab Emirates University, PO Box 17666, Al Ain, UAE; Tel.: + 971-3-7137-516; Fax: + 971-3-7672033;E-mailamine.bahi@uaeu.ac.ae indicating that the D1R pathway within the NAcc may control conditioned-EtOH reward and intake (Bahi and Dreyer, 2012). However, among several observed changes in gene expression upon long-term alcohol consumption, the most consistent alteration was an up-regulation of the dopamine D3 receptors (D3Rs) in the striatum that may contribute to alcohol-seeking and relapse (Vengeliene et al., 2006).

Dopamine is a major neurotransmitter in the central nervous system that plays a critical role in movement, cognition, and emotion (Everitt and Robbins, 2005). The D3R is a member of the D2-like receptor family, which was cloned by Sokoloff and Schwartz (1995), and plays a crucial role in neuropsychiatric disorders (Caine and Koob, 1993), particularly in mediating the addictive properties of alcohol (Boyce and Risinger, 2000). The D3R belongs to class A of G-protein coupled receptors and is primarily localized in brain areas associated with the mesolimbic dopaminergic system related with drug reward (Heidbreder et al., 2004).

Several studies have suggested that selective blockade of the D3R may constitute a useful target for prospective pharmacological therapeutic approaches against alcoholism and to treat other substance use disorders (Newman et al., 2005; Thanos et al., 2005). However, many findings of the effects 
of systemic injection of D3R agonists and antagonists are mixed, with some studies indicating that D3R activation decreases EtOH intake (Silvestre et al., 1996), whereas others find the opposite (Thanos et al., 2005). It is possible that the observed effects are specific to changes in the dopamine in different brain regions and that D3R activation, for example, in the dorsal striatum may have different behavioral effects than D3R activation in other brain regions, such as the NAcc. Nevertheless, recently a role for D3Rs in the modulation of behavioral sensitization by acting as an inhibitor of D1/D2 receptor-mediated behaviors has been proposed (Richtand et al., 2001), and several reports suggest that D3Rs were up-regulated in response to chronic drugs of abuse and this up-regulation contributes to sensitization (Richtand et al., 2001).

More recently, Harrisson and Nobrega (2009) examined EtOH sensitization in D3R knockout (KO) as well as in D1R and D2R KO mice, together with amphetamine sensitization in D3R KOs compared with their wild-type (WT) littermates. They proposed that the development and expression of behavioral sensitization to EtOH is mediated, at least in part by changes in D3R (Harrison and Nobrega, 2009): initially, repeated EtOH administration, through GABAergic dis-inhibition of the mesolimbic pathway, leads to stimulation of D1R in the ventral tegmental area (VTA). Excitation of the VTA leads to increased activity of the mesolimbic pathway that induces an over expression of D3Rs in the NAcc-Shell and ectopic over expression of D3Rs in the dorsal striatum, events that may be transient and mediated only during the induction phase of EtOH sensitization.

After drug cessation, D3Rs in the NAcc remain functionally up-regulated. This functional up-regulation may be long-lasting, and thus reflect at least 1 neural mechanism involved in mediating the expression of EtOH sensitization (Harrison and Nobrega, 2009). In addition, these studies have shown that D3R antagonists do not block the expression of EtOH sensitization once it has already been induced; in contrast, chronic blockade of D3R with an antagonist prevents the induction of EtOH sensitization, but only in individuals that are perhaps genetically more susceptible toward developing sensitization to EtOH (Harrison and Nobrega, 2009).

However, for all these studies, the nonspecificity of ligands and compensatory molecular adaptations in mutated animals restrict the interpretation of the results. To overcome these limitations, lentiviral-mediated gene knockdown and overexpression have been used efficiently to manipulate these genes specifically and locally in the NAcc (Bahi et al., 2005; Fernandes et al., 2012). With this approach, we have shown that D3R expression in the NAcc significantly contributes to behavioral changes associated with chronic cocaine administration: over expression of $\mathrm{D} 3 \mathrm{R}$ in this brain area induces a $50 \%$ decrease in locomotor activity that is reversed to normal upon inactivation of D3R. Furthermore, local knockdown of D3R in the NAcc, efficiently achieved in vivo with lentiviruses expressing D3R-specific small interference RNAs (siRNAs), results in a strong increase in locomotor activity and drug-induced behavioral changes (Bahi et al., 2005).

In the present study, we used pharmacological and genetic approaches to explore the function of the D3R in EtOHrelated behaviors in adult male rats. For this purpose, rats were injected with the D3R antagonist and agonist SB277011-A and 7-OH-DPAT, respectively, and EtOH intake was measured using the 2-bottle choice drinking paradigm. In addition, in a different set of experiments, genetic manipulations were performed to locally knockdown or overexpress the D3R into the NAcc; then, voluntary alcohol intake together with EtOH-conditioned place preference (CPP) behaviors were assessed in Wistar rats. We show that the $\mathrm{D} 3 \mathrm{R}$ is an essential component of the molecular pathways underlying the reinforcing properties of alcohol.

\section{MATERIALS AND METHODS}

\section{Animals}

Adult male Wistar rats (10 weeks) were obtained from the local breeding facility of the College of Medicine \& Health Sciences at $\sim 220 \mathrm{~g}$ of weight and maintained in a temperature-controlled $\left(\sim 22^{\circ} \mathrm{C}\right)$ colony room with a 12 -hour light-dark cycle (0600 to 1800 light on). The experiment began 1 week later at which time the rats were single housed. Bedding was produced locally and autoclaved before use. Water and food were available ad libitum throughout the experiment. Standard rodents' chow diet was obtained from the National Feed and Flour Production and Marketing Company LLC (Abu Dhabi, UAE). The procedures were approved by the United Arab Emirates University, College of Medicine \& Health Sciences Animal Research Ethics Committee (Application Reference No. A27-12).

\section{Drugs}

For CPP experiments, absolute EtOH obtained from Panreac Quimica SAU (Barcelona, Spain) was diluted in isotonic saline $(20 \% ; \mathrm{v} / \mathrm{v})$ and used for intraperitoneal (i.p.) injections at $0.5 \mathrm{~g} /$ $\mathrm{kg}$. The volume of injection was adjusted to body weight. The tastants saccharin sodium salt dihydrate and quinine hemisulfate salt monohydrate were obtained from Sigma-Aldrich (St. Louis, MO). For the 2-bottle choice drinking experiments, EtOH solution was diluted into 2.5 to $20 \%$ solutions (v/v) in tap water. Saccharin $(0.04$ and $0.08 \% ; \mathrm{w} / \mathrm{v})$ and quinine hemisulfate monohydrate (30 and $60 \mu \mathrm{M})$ were dissolved in tap water. The D3R pharmacological agents SB-277011-A hydrochloride hydrate and the 7Hydroxy-2-(di- $n$-propylamino) tetralin hydrobromide (7-OHDPAT) obtained from Sigma-Aldrich were diluted in isotonic saline and doses were adjusted to body weight $(1 \mathrm{ml} / \mathrm{kg})$. Drugs were injected once a day 30 minutes before the light goes off.

\section{Lentiviral-Mediated Overexpression and siRNA Knockdown of D3R}

These vectors were prepared as described previously (Bahi et al., 2005). For more details, see the Supporting Information.

\section{Stereotaxic Microinjection of Lentiviral Vectors}

Stereotaxic injections were performed as described previously (Bahi and Dreyer, 2013; Bahi et al., 2004, 2005). For more details, see the Supporting Information. 


\section{2-Bottle Choice Drinking Paradigm}

Voluntary EtOH consumption and preference was performed as described previously (Bahi, 2012; Bahi and Dreyer, 2013; Bahi et al., 2012). For more details, see the Supporting Information.

\section{Conditioned Place Preference}

The CPP paradigm was selected for the study of lentiviral vectors interactions with EtOH for its procedural simplicity, and the ability to index both the positive and negative motivational properties of alcohol-associated cues. The CPP-test was carried out in a 2-chamber apparatus as described previously (Bahi, 2013b; Bahi and Dreyer, 2013). For more details, see the Supporting Information.

\section{Statistical Analysis}

All analyses were performed using the IBM SPSS package software (version 21.0; Dubai, UAE), and all results were expressed as the mean \pm SEM. The data representing the effect of SB-277011-A and 7-OH-DPAT on EtOH, saccharin, and quinine intake and preference were analyzed using a 1-way analysis of variance (ANOVA) repeated measure with drug as the between-subject factor and time (for EtOH) or concentration (for saccharin and quinine) as the within-subject factor. The data representing the effects of viral injection on $\mathrm{EtOH}$, saccharin, and quinine intakes were analyzed using a 2-way ANOVA repeated measures with virus as the between-subject factor and concentration as the within-subject factor. Data for EtOH-CPP behavior were analyzed using a 1-way ANOVA with virus as the between-subject factor. The Bonferroni test was used for post hoc comparisons of means whenever a significant effect was detected by the ANOVA. Differences were considered significant if $p<0.05$.

\section{RESULTS}

\section{The D3R Antagonist SB-277011-A Attenuated Ethanol Voluntary Consumption}

Figure 1 shows the effects of the injection of saline $(0 \mathrm{mg} /$ $\mathrm{kg}$ ) and increasing doses of SB-277011-A (3, 10, and $30 \mathrm{mg} /$

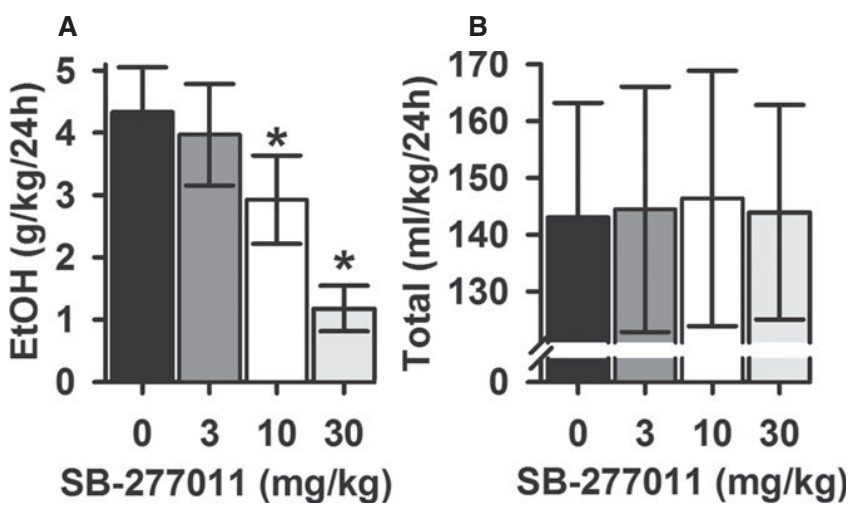

Fig. 1. Voluntary ethanol (EtOH) consumption and total fluid intake following SB-277011-A injection. Wistar rats were injected with increasing doses of the dopamine D3 receptor antagonist SB-277011-A and given access to $5 \% \mathrm{EtOH}$ and water in a 2-bottle choice drinking paradigm for 5 days. (A) Daily $\mathrm{g} / \mathrm{kg}$ EtOH consumption across the experiment. (B) The total fluid intake was the sum of the volume of EtOH solution and water consumed per $\mathrm{kg}$ of body weight per day. The data represent mean \pm SEM. The number of animals per group was $n=8$. $* p<0.05$. $\mathrm{kg})$ on EtOH intake in Wistar rats $(n=8)$. The 1-way ANOVA for repeated measures revealed a significant effect of treatment, $F(3,28)=21.38, p<0.0001$, but not of time, $F(4$, $112)=2.12, p=0.083$. The interaction between time and treatment was significant, $F(12,112)=2.69, \quad p=0.003$ (Fig. 1A). Post hoc comparisons showed that, compared with vehicle, the SB-277011-A doses of 10 and $30 \mathrm{mg} / \mathrm{kg}$ significantly decreased EtOH consumption by $\sim 33 \%$ $(p=0.018)$ and $\sim 73 \%(p<0.0001)$, respectively. However, at $3 \mathrm{mg} / \mathrm{kg}$, the SB-277011-A had no effect on EtOH intake $(p=1.000)$. We also analyzed the effects of SB-277011-A on EtOH preference and as expected there was a significant main effect of treatment, $F(3,28)=48.68, p<0.0001$, and the interaction between time and treatment, $F$ $(12,112)=4.12, \quad p<0.0001 \quad(0 \mathrm{mg} / \mathrm{kg}: \quad 75.72 \% \pm 6.20$; $3 \mathrm{mg} / \mathrm{kg}: \quad 68.26 \% \pm 8.69 ; \quad 10 \mathrm{mg} / \mathrm{kg}: \quad 48.76 \% \pm 8.88$; $30 \mathrm{mg} / \mathrm{kg}: 20.56 \% \pm 5.92$ ). The results for total fluid intake are depicted in Fig. $1 B$. The 1-way ANOVA revealed that there was no main effect of SB-277011-A treatment, $F$ $(3,28)=0.04, p=0.988$, and no significant interaction between time and treatment, $F(12,112)=1.06, p=0.394$. Using the SB-277011-A (30 mg/kg), rats were then tested for taste neophobia using non-EtOH tastants and we found that the D3R antagonist did not affect saccharin and quinine consumption (Fig. S1).

A decreasing effect of the antagonist over trials might indicate development of tolerance with repeated exposure, raising a question about the validity of the later tests. This issue was addressed by exposing the same rats to $5 \%$ EtOH solution and water after quinine intake and as expected, in presence of SB-277011-A (30 mg/kg) rats consumed less alcohol compared to vehicle-injected animals $[1.37 \pm 0.41$ vs. $4.35 \pm 0.56 ; \quad F(1,14)=45.283, \quad p=0.0001]$. Also, SB277011-A injected rats displayed less preference for alcohol compared to vehicle-treated animals $[28.7 \pm 7.99$ vs. $79.4 \pm 5.44 ; F(1,14)=69.526, p=0.0001]$. Finally, there was no difference in total fluid intake between the 2 groups $[136.3 \pm 24.4$ vs. $143.8 \pm 20.6 ; F(1,14)=0.308, p=0.587]$.

\section{Accumbal Knockdown of D3R Decreased Ethanol Consumption and Preference}

To study the possible role of endogenous D3R in the NAcc in the regulation of EtOH intake, we utilized the lentivirus-mediated delivery of siRNA to knockdown the level of the receptor as reported previously (Bahi et al., 2005).

The mean daily intake of EtOH $(\mathrm{g} / \mathrm{kg} / 24 \mathrm{~h})$ for LV-Mock $(n=8)$ and LV-siD3R $(n=8)$ animals was analyzed using a 1-way repeated measures ANOVA, which revealed a significant main effect of virus, $F(1,14)=40.35, p<0.0001$, as well as a main effect of EtOH concentration, $F$ $(3,42)=37.57, p<0.0001$. Interestingly, the interaction between viral injection and EtOH concentration was significant, $F(3,42)=4.01, p=0.013$ (Fig. $2 A$ ). Post hoc evaluations indicated that siD3R-expressing rats consumed less EtOH than Mock controls when 5, 10, and 20\% EtOH 


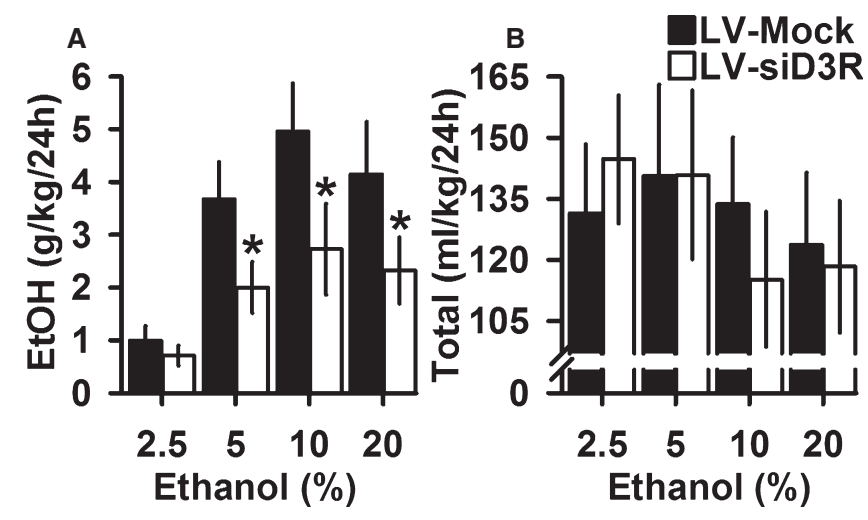

Fig. 2. Voluntary ethanol (EtOH) consumption and total fluid intake following accumbal knockdown of D3R. Wistar rats were stereotaxically injected with LV-Mock $(n=8)$ and LV-siD3R $(n=8)$ into the nucleus accumbens and given access to increasing $\mathrm{EtOH}$ concentrations and water in a 2-bottle choice drinking paradigm for 5 days. (A) Daily g/kg EtOH consumption across the experiment. (B) The total fluid intake was the sum of the volume of $\mathrm{EtOH}$ solution and water consumed per $\mathrm{kg}$ of body weight per day. The data represent mean \pm SEM. $* p<0.05$.

solutions were available $(p<0.001)$. Similarly, a 1-way repeated measure ANOVA also revealed that D3R modulation affected $\mathrm{EtOH}$ preference as evidenced by a significant main effect of viral injection, $F(1,14)=61.80, p<0.0001$, EtOH concentration, $F(3,42)=32.08, p<0.0001$, and a significant main interaction between the 2 factors, $F$ $(3,42)=3.88, p=0.015$ (2.5\%: LV-Mock 34.90\% \pm 7.34 , LV-siD3R 25.48\% \pm 6.18; 5\%: LV-Mock 66.28\% \pm 8.92 , LV-siD3R 36.73\% \pm 7.11; 10\%: LV-Mock 46.24\% \pm 5.32 , LV-siD3R 27.98\% \pm 5.99; 20\%: LV-Mock 22.14\% \pm 5.13 , LV-siD3R 14.50\% \pm 4.63). Finally, LV-Mock and LVsiD3R were tested for total fluid intake along the entire 2bottle choice paradigm using a 2-way ANOVA repeated measure. As depicted in Fig. 2B, our results showed that no difference between siD3R-expressing rats and control animals were observed in mean fluid intake, main viral injection, $F(1,14)=0.93, p=0.351$, main effect of EtOH concentration, $F(3,42)=0.92, p=0.438$, and the interaction between the 2 variables, $F(3,42)=0.28, p=0.834$ (Fig. $2 B)$. To control for any taste neophobia following D3R knockdown in the NAcc, the same rats were tested with increasing concentrations of saccharin and quinine. Results have shown that accumbal knockdown of D3R had no effect on saccharin and quinine intake and preference (Fig. S2).

\section{The D3R Agonist 7-OH-DPAT Increased Ethanol Voluntary Intake in Rats}

In the following set of experiments, we investigated the effects of the D3R selective agonist 7-OH-DPAT $(0.05 \mathrm{mg} /$ $\mathrm{kg}$ ) on voluntary alcohol consumption in Wistar rats. Dosing and preapplication of 7-OH-DPAT was conducted based on previously published studies, where 0.01 to $0.5 \mathrm{mg} / \mathrm{kg}$ of $7-$ OH-DPAT were used in both rats (Depoortere et al., 1996; Frantz et al., 1996; Gainetdinov et al., 1996) and mice
(Ferrari and Giuliani, 1997; Kamei and Saitoh, 1996; Starr and Starr, 1995).

We compared EtOH consumption of vehicle- $(0 \mathrm{mg} / \mathrm{kg}$; $n=8)$ and 7-OH-DPAT- $(0.05 \mathrm{mg} / \mathrm{kg} ; n=8)$ injected rats in a 2-bottle choice alcohol-drinking paradigm and results are shown in Fig. 3A. A 1-way ANOVA analysis indicated that 7-OH-DPAT-injected rats showed significantly high EtOH intake, $F(1,14)=14.76, p=0.002$. Similarly, EtOH preference was also increased following 7-OH-DPAT injection, $F$ $(1,14)=80.63, \quad p<0.0001 \quad(0 \mathrm{mg} / \mathrm{kg}: \quad 67.04 \% \pm 6.53$; $0.05 \mathrm{mg} / \mathrm{kg}: 92.89 \% \pm 2.92)$. Interestingly, and as shown in Fig. $3 B$, there was no difference in total fluid intake between the 2 experimental groups, $F(1,14)=0.24, p=0.626$. Interestingly, when tested for saccharin and quinine intake, the D3R agonist 7-OH-DPAT had no effect on taste neophobia (Fig. S3).

\section{Accumbal D3R Overexpression Increased Voluntary Ethanol Consumption}

In this experiment, control vectors (LV-Mock; $n=8$ ) and D3R-overexpressing lentiviral vectors (LV-D3R; $n=8$ ) were injected into the NAcc and EtOH intake was monitored using the 2-bottle choice drinking paradigm as described in the Materials and Methods section above. Results are depicted in Fig. 4. The 1-way ANOVA revealed that LVD3R-expressing rats displayed increased EtOH intake from the 5, 10 and $20 \%$ drinking solutions. In fact, there was a significant effect of viral injection, $F(1,14)=35.04, p<0.0001$, and concentration, $F(3,42)=70.45, p<0.0001$, on EtOH consumption. Interestingly, the virus $\times \mathrm{EtOH}$ interaction was significant, $F(3,42)=3.89, p=0.015$ (Fig. 4A). We then assessed the effect of D3R overexpression on EtOH preference and the 1-way ANOVA analysis revealed a main effect of viral injection, $F(1,14)=79.39, p<0.0001$, a main
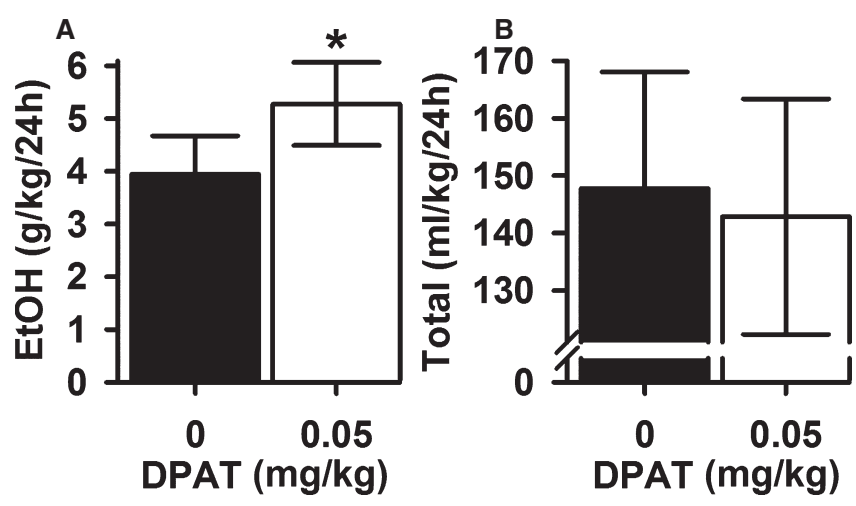

Fig. 3. Voluntary ethanol $(\mathrm{EtOH})$ consumption and total fluid intake following 7-OH-DPAT injection. Wistar rats were injected with the vehicle or the D3R agonist 7-OH-DPAT $(0.05 \mathrm{mg} / \mathrm{kg})$ and given access to $5 \% \mathrm{EtOH}$ and water in a 2-bottle choice drinking paradigm for 5 days. (A) Daily g/kg EtOH consumption across the experiment. (B) The total fluid intake was the sum of the volume of $\mathrm{EtOH}$ solution and water consumed per $\mathrm{kg}$ of body weight per day. The data represent mean \pm SEM. The number of animals per group was $n=8$. * $p<0.05$. 


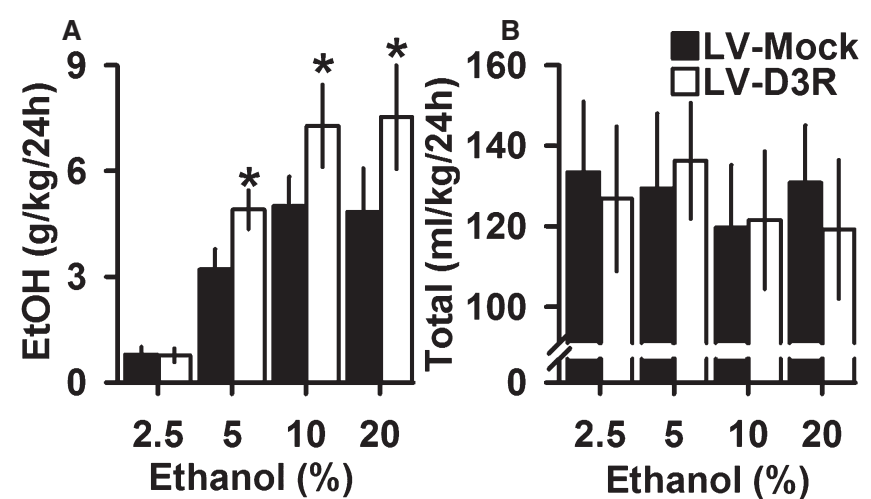

Fig. 4. Voluntary ethanol (EtOH) consumption and total fluid intake following accumbal overexpression of D3R. Wistar rats were stereotaxically injected with LV-Mock $(n=8)$ and LV-D3R $(n=8)$ into the nucleus accumbens and given access to increasing $\mathrm{EtOH}$ concentrations and water in a 2-bottle choice drinking paradigm for 5 days. (A) Daily g/kg EtOH consumption across the experiment. (B) The total fluid intake was the sum of the volume of EtOH solution and water consumed per $\mathrm{kg}$ of body weight per day. The data represent mean \pm SEM. $* p<0.05$

effect of EtOH concentration, $F(3,42)=106.45, p<0.0001$, as well as a significant interaction between the 2 factors, $F$ $(3,42)=4.71, p=0.006(2.5 \%$ : LV-Mock 28.90\% \pm 6.05 , LV-D3R $31.04 \% \pm 7.14 ; 5 \%$ : LV-Mock $65.97 \% \pm 9.24$, LV-D3R 90.75\% \pm 3.16; 10\%: LV-Mock 52.86\% \pm 6.34 , LV-D3R $73.955 \pm 5.31 ; 20 \%$ : LV-Mock 23.64\% \pm 5.45 , LV-D3R $41.83 \% \pm 6.25)$. Finally, the total liquid consumption did not differ between the 2 experimental groups indicating normal drinking behavior in LV-D3R-injected rats; main effect of virus, $F(1,14)=0.25, p=0.619$; main effect of EtOH concentration, $F(3,42)=0.92, p=0.436$; main effect of virus $\times$ EtOH interaction, $F(3,42)=0.53, p=0.660$ (Fig. 4B). To determine whether any difference existed in general taste preferences, drinking studies using saccharin and quinine were performed after the EtOH drinking study. Results have shown that accumbal D3R overexpression had no effect on saccharin and quinine intake and preference (Fig. S4).

\section{Viral-Mediated Modulation of D3R Impairs EtOH-CPP}

EtOH can establish preference for an environment associated with repeated drug exposure. The CPP paradigm serves as a model of rewarding and abusive effects of repeated EtOH administration (Reid et al., 1985). In this experiment, animals were conditioned with repeated EtOH injection in 1 of 2 chambers that differed visually and texturally and results are depicted in Fig. 5. As was expected, the administration of $0.5 \mathrm{~g} / \mathrm{kg}$ of EtOH in rats produced CPP. In our laboratory, this result has been previously observed in different studies using the same experimental procedure (Bahi, 2012; Bahi and Dreyer, 2013). Therefore, before conditioning, the LV-Mock $(n=8)$, LV-siD3R $(n=8)$, and LV-D3R $(n=8)$ experimental groups visited the 2 chambers, and no main effect of virus was detected, $F(2,21)=0.59, p=0.562$. However, after conditioning with repeated EtOH exposure, the

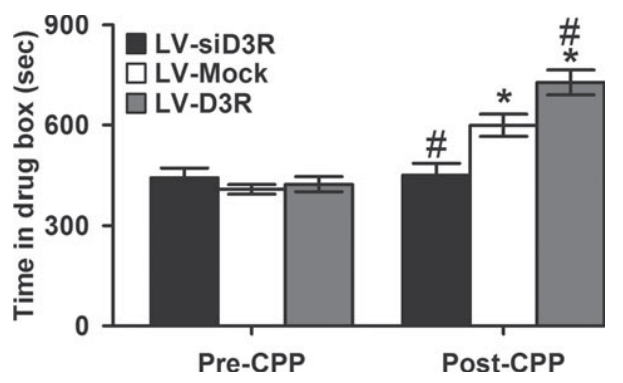

Fig. 5. Ethanol (EtOH)-induced conditioned place preference (CPP) following lentiviral-mediated modulation of D3R into the nucleus accumbens. Histograms show the mean \pm SEM of the time spent into the EtOHpaired chamber during the pre- and the postconditioning sessions. After baseline testing (Pre-CPP), animals were stereotaxically injected with LVMock $(n=8)$, LV-siD3R $(n=8)$, or LV-D3R $(n=8)$. After recovery, animals were conditioned with either saline or $\mathrm{EtOH}$, and then tested for their preference (Post-CPP). $* p<0.05$ compared to the corresponding groups during Pre-CPP, $\# p<0.05$ compared to LV-Mock during the Post-CPP.

1-way ANOVA revealed a main effect of viral injection on CPP scores, $F(2,21)=15.48, p<0.0001$. Post hoc evaluations indicated that LV-D3R-overexpressing rats showed $\sim 21 \%$ enhancement of EtOH-CPP $(p=0.053)$. In contrast and compared to LV-Mock, D3R-knockdown displayed $\sim 25 \%$ decrease of EtOH-CPP $(p=0.021)$. The results indicate that D3R in the NAcc plays an important role in EtOHconditioned reward-associated learning.

During the CPP-test, viral injection had no effect on spontaneous locomotor activity and results are depicted in Fig. S5. After completion of the CPP experiments, rats D3R mRNA was quantified by reverse transcription polymerase chain reaction and results are displayed in Fig. S6. The histological representation of injection placements in the NAccShell are summarized in Fig. S7.

\section{DISCUSSION}

Previous work from our laboratory have shown a direct relationship between the D3R and cocaine-induced locomotor stimulation, such that NAcc-Shell knockdown of D3R using specific small hairpin RNA (shRNA) showed strong cocaine-induced locomotor stimulation in rats (Bahi et al., 2005). Although the relationship between the D3R system and EtOH-related behaviors has been demonstrated in a number of studies, it is not known how D3R neurotransmission relates to preference for EtOH. In this study, we addressed this issue using EtOH consumption and preference in a 2-bottle choice test as well as EtOH-CPP. We show that pharmacological inhibition of the D3R inhibited voluntary alcohol consumption and preference. In contrast, the D3R agonist 7-OH-DPAT exacerbated $\mathrm{EtOH}$ intake in Wistar rats. More importantly, lentiviral-mediated knockdown of the D3R into the NAcc blocked EtOH intake as well as EtOH-CPP. However, D3R-overexpression in the same brain region increased both voluntary EtOH intake and preference together with EtOH-CPP. Interestingly, control experiments revealed that nonalcoholic tastants (saccharin 
and quinine) consumption was not affected following D3R lentiviral-mediated loss- and gain-of-function. These data are the first to show a causal link between accumbal D3R and the motivational effects of EtOH. Based on these results, we hypothesize that D3R is a key regulator of voluntary EtOH intake and EtOH-induced conditioned reward.

CPP is a behavioral procedure allowing measuring the rewarding effects of a drug. Although EtOH-induced CPP is a common finding in mice, the literature on $\mathrm{EtOH}$ reward using CPP procedure has generated conflicting results in rats. For example, Ciccocioppo and colleagues (1999) reported that in naïve rats, only $0.7 \mathrm{~g} / \mathrm{kg}$ EtOH significantly increased the time spent in the EtOH-associated chamber. In contrast, Asin and colleagues (1985) have shown that the i.p. administration of a wide range of EtOH concentrations $(0.05,0.1$, $0.15,0.3,0.6,0.8$, or $1 \mathrm{~g} / \mathrm{kg}$ ) failed to produce either a CPP or aversion compared to vehicle injected control rats. Also, Quertemont and De Witte (2001) observed that EtOH injections mainly induced aversive effects with doses above $1 \mathrm{~g} /$ $\mathrm{kg}$, while no reinforcing effects were observed for lower 0.25 and $0.5 \mathrm{~g} / \mathrm{kg}$ EtOH doses. On the other hand, it has been shown that after receiving a total of 15 daily conditioning trials under $1 \mathrm{~g} / \mathrm{kg} \mathrm{EtOH}$, a significant place preference was produced (Bozarth, 1990). Therefore, the rewarding effects of EtOH may be due to a complex interaction among a variety of factors including genetics, route and dose of administration, and number and time of conditioning trials (for review, see Tzschentke, 2007).

In the first set of experiments, we showed that pharmacological blockade and genetic knockdown, using SB-277011A and LV-siD3R, respectively, effectively reduced voluntary EtOH intake and preference and EtOH-CPP-acquisition. This supports previous studies showing that D3R antagonism, using SB-277011-A, selectively reduces EtOH intake. In fact, it has been reported that in alcohol-Preferring $(\mathrm{P})$ and Non-Preferring (NP) rats, there was a significant decrease in alcohol consumption and preference in $\mathrm{P}$ rats treated with 10 and $30 \mathrm{mg} / \mathrm{kg} \mathrm{SB}-277011-\mathrm{A}$ (Thanos et al., 2005). In addition, Vengeliene and colleagues (2006) reported that after 1 year of voluntary alcohol consumption, DNA microarray analysis revealed an up-regulation of the D3R in the striatum of $\mathrm{P}$ rats as well as in nonselected regular Wistar rats after long-term alcohol consumption. More importantly, in the same study, the authors found that the selective D3R antagonist SB-277011-A caused a dose-dependent reduction of relapse-like drinking in the alcohol deprivation effect model as well as a decrease in cue-induced EtOH-seeking behavior (Vengeliene et al., 2006). Our data are also in agreement with previous studies demonstrating that SB-277011-A was effective in reducing drug craving and relapse to other abused substances (Di Ciano et al., 2003; Gilbert et al., 2005; Le Foll et al., 2002).

Several other studies using D3R KO mice were inconclusive. In fact, when examining the development of physical dependence on $\mathrm{EtOH}$ in WT and $\mathrm{KO}$ mice following chronic treatment with $7 \% \mathrm{EtOH}$, severe withdrawal signs were observed in D3R-KO as compared with WT. In addition, D3R KO mice (on a C57 background from the Jackson Laboratory, Bar Harbor, ME) display increased initial sensitivity to $\mathrm{EtOH}$ as measured by an increase in sleep time to a $3.5 \mathrm{~g} /$ $\mathrm{kg}$ dose of $\mathrm{EtOH}$ (Narita et al., 2002) suggesting that the lack of D3R leads to the aggravation of the development of physical dependence on EtOH. However, another transgenic $\mathrm{KO}$ approach concluded that total ablation of the D3R had little influence on EtOH intake. In fact, EtOH-CPP magnitudes were similar in both genotypes. Also, in a 2-bottle drinking procedure, no difference was noted between D3R KO and their WT. Finally, in an operant self-administration procedure both groups of mice had similar response rates of EtOH and food as well as similar water intakes (Boyce-Rustay and Risinger, 2003) indicating that total genetic ablation of the D3R has little influence on alcohol intake or reward.

In our study, both pharmacological and genetic blockade of the D3R produced similar effects on voluntary alcohol intake and preference. More importantly, selective genetic blockade of D3R into the NAcc blocked EtOH-CPP-acquisition. This suggests that in the presence of environmental cues previously associated with $\mathrm{EtOH}$, which would induce mesolimbic dopamine release, D3R knockdown, using shRNAexpressing lentiviral vectors, might be acting as a D3R antagonist. This is in line with our previous finding that these viral vectors were efficient to reduce D3R expression both at the mRNA and protein levels (Bahi et al., 2005). From these data, we therefore believe that selective D3R antagonism and lentiviral-mediated genetic shRNA blockade impairs EtOH-related behaviors by modulating the activity of midbrain dopaminergic neurons and hence voluntary EtOH intake and conditioned reward.

In the second set of experiments, we found that the D3R pharmacological and genetic activation, using the agonist 7OH-DPAT and D3R-overexpressing lentiviral vectors respectively, increased voluntary EtOH intake as well as EtOH-CPP. Our results support previous findings showing that D3R agonism, using 7-OH-DPAT, at the lowest dose of $0.01 \mathrm{mg} / \mathrm{kg}$ increased both EtOH intake and preference in rats with access to an EtOH solution (10\% EtOH, 3\% glucose) (Silvestre et al., 1996). In contrast, it has been reported that when rats were trained to self-administer EtOH $(10 \%$, $\mathrm{v} / \mathrm{v}$ ) orally in a free-choice 2-lever operant task, 7-OH-DPAT $(0.003-0.1 \mathrm{mg} / \mathrm{kg})$ resulted in dose-dependent decreases in responding for EtOH (Cohen et al., 1998). In the same line, when mice were given EtOH, 7-OH-DPAT (0.5 and $1 \mathrm{mg} /$ $\mathrm{kg})$ blocked the EtOH $(0.5 \mathrm{~g} / \mathrm{kg})$-induced locomotor hyperactivity dose dependently and at doses that did not affect locomotor activity in naïve mice when administered alone (Uzbay and Kayir, 2003). It should be emphasized that D3R lentiviral-mediated overexpression had no effect on locomotor activity during the CPP-test session. Therefore, it is unlikely that the alterations in CPP (observed in LV-D3Rexpressing rats) were a byproduct of lentiviral vector effects on activity. Nevertheless, one weakness of lentiviral approach used in this study is that effects on EtOH-induced 
CPP cannot be isolated to either the acquisition phase (when EtOH is present) or test phase (in the absence of EtOH). Therefore, future experiments with viral vectors injected after conditioning are of great interest to tease apart whether D3R is involved in CPP-acquisition or CPP-expression.

Finally, a taste neophobia analysis of saccharin and quinine revealed that rats with both pharmacological and genetic blockade of the D3R consume comparable amounts of saccharin and quinine. Unlike most other drugs of abuse, alcohol must be consumed orally and therefore it was important to test how the animals perceive taste. To assess this issue, rats were exposed to different concentrations of both a sweet and a bitter solution. These solutions were chosen because they have been demonstrated previously to detect taste sensitivity, lack calories, and have palatable differences in both mice and rats (Bahi, 2012, 2013a; Bahi and Dreyer, 2012; Bahi et al., 2013). Results have shown that there were no differences in consumption or preference between the different groups. These data suggest that rats treated with vehicle, SB-277011-A and 7-OH-DPAT or stereotaxically injected into the NAcc with LV-Mock, LV-siD3R, or LVD3R had comparable sensory function and that the results found with $\mathrm{EtOH}$ are not due to a deficit in taste processing, at least in Wistar rats.

Our current study, utilizing alternative molecular methods (specific shRNA-expressing lentiviral vectors), addressed concerns about the influence of possible developmental changes as well as functional compensations seen with the D3R KO used in the above-mentioned studies and the role of the D3R in alcohol-related behaviors. Our experiments utilizing both genetic and pharmacological blockade were conducted to resolve the contribution of brain regions, strain, genetic background, and pharmacological manipulation in the role of D3R in voluntary alcohol consumption and EtOH-CPP. In the present study, NAcc-D3R knockdown reduced EtOH intake and place preference, whereas results obtained with $\mathrm{D} 3 \mathrm{R} \mathrm{KO}$ mice did not show altered EtOH-induced CPP (possibly due to functional compensations). Our data clearly shows that D3R, indeed, plays a critical role in EtOH-induced behaviors. Also, determination of the effect of pharmacological and genetic blockade on EtOH-induced CPP was needed for a more complete understanding of the failure of D3R KO mice to show impaired EtOH intake and EtOH-CPP. Infusion of lentiviral vectors expressing siRNAs targeting D3R directly into the NAcc blocked or attenuated the formation of CPP in adult Wistar rats. These behavioral changes are likely to be due to knockdown of D3R mRNA resulting in decreased receptor density at the infusion site demonstrating the utility of siRNAexpressing viral vectors in the neurobiological study of specific components of the reward system.

In conclusion, the present study elucidates how D3R in anatomically specific manner mediate voluntary alcohol intake and preference and $\mathrm{EtOH}-\mathrm{CPP}$, findings that can be explored in other preclinical rodent models of addiction in general and alcoholism in particular. Finally, we have provided a new sight in molecular mechanism on the effect of D3R in alcohol intake.

\section{DISCLOSURE/CONFLICT OF INTEREST}

The authors declare no conflict of interests. AB is receiving salary and funds from the United Arab Emirates University. The funder had no role in study design, data collection and analysis, decision to publish, or preparation of the manuscript.

\section{ACKNOWLEDGMENTS}

The authors would like to acknowledge Ms. Christine Deforel-Poncet, Mr. Mohamed Elwasila, and Mr. Mohamed Shafiullah for their technical assistance, and Dr. Mahmoud Hag Ali from the Central Animal Facility for his advice on veterinary care.

\section{REFERENCES}

Asin KE, Wirtshafter D, Tabakoff B (1985) Failure to establish a conditioned place preference with ethanol in rats. Pharmacol Biochem Behav 22:169-173.

Bahi A (2012) Viral-mediated knockdown of mGluR7 in the nucleus accumbens mediates excessive alcohol drinking and increased ethanol-elicited conditioned place preference in rats. Neuropsychopharmacology 38:21092119.

Bahi A (2013a) Individual differences in elevated plus-maze exploration predicted higher ethanol consumption and preference in outbred mice. Pharmacol Biochem Behav 105:83-88.

Bahi A (2013b) Viral-mediated knockdown of mGluR7 in the nucleus accumbens mediates excessive alcohol drinking and increased ethanol-elicited conditioned place preference in rats. Neuropsychopharmacology $38: 2109-2119$.

Bahi A, Boyer F, Bussard G, Dreyer JL (2005) Silencing dopamine D3-receptors in the nucleus accumbens shell in vivo induces changes in cocaineinduced hyperlocomotion. Eur J Neurosci 21:3415-3426.

Bahi A, Boyer F, Kafri T, Dreyer JL (2004) CD81-induced behavioural changes during chronic cocaine administration: in vivo gene delivery with regulatable lentivirus. Eur J Neurosci 19:1621-1633.

Bahi A, Dreyer JL (2012) Involvement of nucleus accumbens dopamine D1 receptors in ethanol drinking, ethanol-induced conditioned place preference, and ethanol-induced psychomotor sensitization in mice. Psychopharmacology 222:141-153.

Bahi A, Dreyer JL (2013) Striatal modulation of BDNF expression using microRNA124a-expressing lentiviral vectors impairs ethanol-induced conditioned-place preference and voluntary alcohol consumption. Eur J Neurosci 38:2328-2337.

Bahi A, Fizia K, Dietz M, Gasparini F, Flor PJ (2012) Pharmacological modulation of mGluR7 with AMN082 and MMPIP exerts specific influences on alcohol consumption and preference in rats. Addict Biol 17:235247.

Bahi A, Tolle V, Fehrentz JA, Brunel L, Martinez J, Tomasetto CL, Karam SM (2013) Ghrelin knockout mice show decreased voluntary alcohol consumption and reduced ethanol-induced conditioned place preference. Peptides 43C:48-55.

Boyce JM, Risinger FO (2000) Enhancement of ethanol reward by dopamine D3 receptor blockade. Brain Res 880:202-206.

Boyce-Rustay JM, Risinger FO (2003) Dopamine D3 receptor knockout mice and the motivational effects of ethanol. Pharmacol Biochem Behav 75:373-379. 
Bozarth MA (1990) Evidence for the rewarding effects of ethanol using the conditioned place preference method. Pharmacol Biochem Behav 35:485487.

Caine SB, Koob GF (1993) Modulation of cocaine self-administration in the rat through D-3 dopamine receptors. Science 260:1814-1816.

Ciccocioppo R, Panocka I, Froldi R, Quitadamo E, Massi M (1999) Ethanol induces conditioned place preference in genetically selected alcohol-preferring rats. Psychopharmacology 141:235-241.

Cohen C, Perrault G, Sanger DJ (1998) Preferential involvement of D3 versus D2 dopamine receptors in the effects of dopamine receptor ligands on oral ethanol self-administration in rats. Psychopharmacology 140:478-485.

Depoortere R, Perrault G, Sanger DJ (1996) Behavioural effects in the rat of the putative dopamine D3 receptor agonist 7-OH-DPAT: comparison with quinpirole and apomorphine. Psychopharmacology 124:231-240.

Di Ciano P, Underwood RJ, Hagan JJ, Everitt BJ (2003) Attenuation of cuecontrolled cocaine-seeking by a selective D3 dopamine receptor antagonist SB-277011-A. Neuropsychopharmacology 28:329-338.

Everitt BJ, Robbins TW (2005) Neural systems of reinforcement for drug addiction: from actions to habits to compulsion. Nat Neurosci 8:14811489.

Fernandes AR, Easton AC, De Souza Silva MA, Schumann G, Muller CP, Desrivieres $S$ (2012) Lentiviral-mediated gene delivery reveals distinct roles of nucleus accumbens dopamine D2 and D3 receptors in novelty- and light-induced locomotor activity. Eur J Neurosci 35:1344-1353.

Ferrari F, Giuliani D (1997) Effects of (-)eticlopride and 7-OH-DPAT on the tail-suspension test in mice. J Psychopharmacol 11:339-344.

Frantz K, Babcock D, Van Hartesveldt C (1996) The locomotor effects of a putative dopamine D3 receptor agonist in developing rats. Eur J Pharmacol 302:1-6.

Gainetdinov RR, Sotnikova TD, Grekhova TV, Rayevsky KS (1996) In vivo evidence for preferential role of dopamine D3 receptor in the presynaptic regulation of dopamine release but not synthesis. Eur J Pharmacol 308:261-269.

Gilbert JG, Newman AH, Gardner EL, Ashby CR Jr, Heidbreder CA, Pak AC, Peng XQ, Xi ZX (2005) Acute administration of SB-277011A, NGB 2904, or BP 897 inhibits cocaine cue-induced reinstatement of drug-seeking behavior in rats: role of dopamine D3 receptors. Synapse 57:17-28.

Harrison SJ, Nobrega JN (2009) A functional role for the dopamine D3 receptor in the induction and expression of behavioural sensitization to ethanol in mice. Psychopharmacology 207:47-56.

Heidbreder CA, Andreoli M, Marcon C, Thanos PK, Ashby CR Jr, Gardner EL (2004) Role of dopamine D3 receptors in the addictive properties of ethanol. Drugs Today (Barc) 40:355-365.

Kamei J, Saitoh A (1996) Modification of the effects of 7-OH-DPAT, a dopamine D3-receptor agonist, on morphine-induced hyperlocomotion by diabetes. Life Sci 59:773-780.

Le Foll B, Frances H, Diaz J, Schwartz JC, Sokoloff P (2002) Role of the dopamine D3 receptor in reactivity to cocaine-associated cues in mice. Eur J Neurosci 15:2016-2026.

Narita M, Soma M, Tamaki H, Narita M, Suzuki T (2002) Intensification of the development of ethanol dependence in mice lacking dopamine $\mathrm{D}(3)$ receptor. Neurosci Lett 324:129-132.

Newman AH, Grundt P, Nader MA (2005) Dopamine D3 receptor partial agonists and antagonists as potential drug abuse therapeutic agents. J Med Chem 48:3663-3679.

Quertemont E, De Witte P (2001) Conditioned stimulus preference after acetaldehyde but not ethanol injections. Pharmacol Biochem Behav 68:449-454.
Reid LD, Hunter GA, Beaman CM, Hubbell CL (1985) Toward understanding ethanol's capacity to be reinforcing: a conditioned place preference following injections of ethanol. Pharmacol Biochem Behav 22:483-487.

Richtand NM, Goldsmith RJ, Nolan JE, Berger SP (2001) The D3 dopamine receptor and substance dependence. J Addict Dis 20:19-32.

Silvestre JS, O'Neill MF, Fernandez AG, Palacios JM (1996) Effects of a range of dopamine receptor agonists and antagonists on ethanol intake in the rat. Eur J Pharmacol 318:257-265.

Sokoloff P, Schwartz JC (1995) Novel dopamine receptors half a decade later. Trends Pharmacol Sci 16:270-275.

Spanagel R, Heilig M (2005) Addiction and its brain science. Addiction 100:1813-1822.

Spanagel R, Weiss F (1999) The dopamine hypothesis of reward: past and current status. Trends Neurosci 22:521-527.

Starr MS, Starr BS (1995) Motor actions of 7-OH-DPAT in normal and reserpine-treated mice suggest involvement of both dopamine D2 and D3 receptors. Eur J Pharmacol 277:151-158.

Thanos PK, Katana JM, Ashby CR Jr, Michaelides M, Gardner EL, Heidbreder CA, Volkow ND (2005) The selective dopamine D3 receptor antagonist SB-277011-A attenuates ethanol consumption in ethanol preferring (P) and non-preferring (NP) rats. Pharmacol Biochem Behav 81:190-197.

Tzschentke TM (2007) Measuring reward with the conditioned place preference (CPP) paradigm: update of the last decade. Addict Biol 12: $227-462$.

Uzbay IT, Kayir H (2003) Bromocriptine and quinpirole, but not 7-OHDPAT or SKF 38393, potentiate the inhibitory effect of L-NAME on ethanol-induced locomotor activity in mice. Naunyn Schmiedebergs Arch Pharmacol 367:414 421.

Vengeliene V, Leonardi-Essmann F, Perreau-Lenz S, Gebicke-Haerter P, Drescher K, Gross G, Spanagel R (2006) The dopamine D3 receptor plays an essential role in alcohol-seeking and relapse. FASEB J 20:2223-2233.

\section{SUPPORTING INFORMATION}

Additional Supporting Information may be found in the online version of this article:

Data S1. Materials and Methods.

Fig. S1. Voluntary saccharin and quinine consumption and total fluid intake following SB-277011-A injection.

Fig. S2. Saccharin and quinine consumption and total fluid intake following accumbal knockdown of D3R.

Fig. S3. Voluntary saccharin and quinine consumption and total fluid intake following 7-OH-DPAT injection.

Fig. S4. Saccharin and quinine consumption and total fluid intake following accumbal overexpression of D3R.

Fig. S5. Effects of D3R overexpression and knockdown on locomotors activity during the CPP-test.

Fig. S6. Effects of D3R overexpression and knockdown on mRNA expression.

Fig. S7. Histological representation of injection placements in the NAcc-Shell. 